Revista Arbitrada Interdisciplinaria KOINONIA

Año VI. Vol VI. N³. Edición Especial: Educación II. 2021

Hecho el depósito de Ley: FA2016000010 ISSN: 2542-3088

FUNDACIÓN KOINONIA (F.K). Santa Ana de Coro. Venezuela.

Katherine Michelle Zhindón-Calle; Carlos Marcelo Ávila-Mediavilla

http://dx.doi.org/10.35381/r.k.v6i3.1303

\title{
Tecnologías emergentes aplicadas a la práctica educativa en pandemia COVID-19
}

\section{Emerging technologies applied to educational practice in pandemic COVID-19}

\author{
Katherine Michelle Zhindón-Calle \\ katherine.zhindon.13@est.ucacue.edu.ec \\ Universidad Católica de Cuenca, Azogues \\ Ecuador \\ https://orcid.org/0000-0002-5497-0382 \\ Carlos Marcelo Ávila-Mediavilla \\ cavilam@ucacue.edu.ec \\ Universidad Católica de Cuenca, Cuenca \\ Ecuador \\ https://orcid.org/0000-0002-2649-9634
}

Recepción: 10 de abril 2021

Revisado: 05 de mayo 2021

Aprobación: 30 de junio 2021

Publicación: 15 de julio 2021 


\title{
RESUMEN
}

El objetivo de la investigación fue analizar el uso de los recursos tecnológicos en la práctica educativa durante la pandemia COVID-19, mediante el análisis de la formación docente y el empleo de los distintos recursos digitales. Se realizó una investigación de tipo descriptivo no experimental de cohorte transversal, con un paradigma epistemológico cuantitativo, se aplicó una encuesta online a todos los docentes y a los estudiantes de los niveles de educación básica y superior de la Escuela de Educación Básica Gabriela Mistral. Es así como se puede notar que los docentes a pesar de no haber recibido capacitación necesaria sobre las tecnologías emergentes y no sentirse preparados para el cambio drástico de las clases presenciales a la práctica educativa en la virtualidad hicieron uso de distintos recursos tecnológicos implementándolos en la práctica educativa con el fin de innovar las clases para motivar a los estudiantes y generar aprendizajes significativos.

Descriptores: Estrategias educativas; tecnología educacional; informática educativa. (Palabras tomadas del Tesauro UNESCO).

\begin{abstract}
The objective of the research was to analyze the use of technological resources in educational practice during the COVID-19 pandemic, by analyzing teacher training and the use of different digital resources. A descriptive, non-experimental cross-sectional cohort research was carried out, with a quantitative epistemological paradigm, an online survey was applied to all teachers and students of the basic and higher education levels of the Gabriela Mistral School of Basic Education. This is how it can be seen that teachers, despite not having received the necessary training on emerging technologies and not feeling prepared for the drastic change from face-to-face classes to educational practice in virtuality, made use of different technological resources implementing them in practice. educational in order to innovate classes to motivate students and generate meaningful learning.
\end{abstract}

Descriptors: Educational strategies; educational technology; computer uses in education. (Words taken from the UNESCO Thesaurus). 


\section{INTRODUCCIÓN}

En la ciudad de Wuhan en China en el mes de diciembre de 2019, se dio un brote de casos de una neumonía grave tras varias investigaciones se determinó que se trataba de un agente viral nuevo, y fue llamado COVID-19 (Coronavirus disease-2019), durante los primeros meses del 2020 el brote se propagó a otros países y continentes. A partir del mes de marzo la vida como la conocíamos cambio drásticamente, la Organización Mundial de la Salud debido a los altos niveles de propagación de la enfermedad y por su gravedad, la determino, como una pandemia esta noticia se esparció en todos los noticieros a nivel mundial.

Ante este suceso el mundo entro en alerta y se determinó un confinamiento total, como medida de contención en la cual se quería reducir las interacciones sociales para evitar los posibles contagios; espacios públicos como parques, cines, centros comerciales, oficinas, escuelas etc; se cerraron por completo, varios sectores se vieron afectados con el confinamiento obligatorio, por su puesto la educación no fue la excepción.

Las escuelas fueron cerradas totalmente, estudiantes y docentes debían permanecer en su casa para precautelar la salud y seguridad, la actividad educativa presencial se suspendió, para continuar con el proceso educativo se buscó otras alternativas generando una educación a distancia en todos los niveles educativos a través de medios tecnológicos como la televisión, radio, celulares, computadores; para muchas personas las medidas tomadas como alternativas no fueron accesibles debido a varias situaciones como: el desconocimiento del uso de los recursos tecnológicos, la falta de conectividad o la escasees de posibilidades para acceder a los mismos (De-Giusti, 2020).

De acuerdo con (Palomeque \& Parrales, 2021), la pandemia generó un cambio drástico en la educación, dejamos atrás las formas tradicionales a las cuales estábamos acostumbrados, los procesos educativos, la relación alumno - maestro, las clases en el aula, los juegos en el patio, las rutinas diarias de la presencialidad con el fin de adaptarnos a la nueva normalidad haciendo uso de los recursos tecnológicos como fuente mediadora para realizar en lo posible algunos de estos procesos. 
Adoptamos el termino educación a distancia para darle un nombre a la actividad emergente que realizamos desde entonces hasta la actualidad, sin embargo es necesario entender que la educación a distancia ya tiene sus antecedentes mucho antes de esta pandemia, es un modalidad educativa la cual ya cuenta con un sustento y diseño pedagógico y por su puesto docentes capacitados en esta practica.

En este sentido, (Singh \& Thurman, 2019), definen la educación a distancia como la actividad que se realiza en un entorno on-line a través del uso de Internet para el proceso de enseñanza-aprendizaje, no depende de su ubicación física o virtual. El contenido de la enseñanza se entrega en línea y los instructores desarrollan módulos de enseñanza que mejoran el aprendizaje y la interactividad en el entorno sincrónico o asincrónico.

En Ecuador a partir del 12 de marzo del 2020 el Gobierno Nacional dispuso la suspensión de las clases presenciales indefinidamente a nivel nacional en escuelas, colegios, universidades, considerando que para el Estado ecuatoriano conforme al Art. 26 de la (Constitución de la República del Ecuador, 2008), la educación es un derecho fundamental de las personas a lo largo de su vida, se considera una área prioritaria e indispensable en la cual se garantiza la igualdad e inclusión social para el buen vivir; con el fin de dar continuidad a la práctica educativa docentes y estudiantes se vieron en la necesidad de hacer uso de los recursos tecnológicos como medio de comunicación y de formación, al inicio las indicaciones y tareas se envían a través de redes sociales como WhatsApp, Facebook, y las clases se dictaban a través de videollamadas.

El Ministerio de Educación (MINEDUC, 2020) creo la plataforma virtual Plan Educativo COVID-19, la que fue creada con el fin de reforzar los aprendizajes ya adquiridos; esta plataforma está conformado con más de 2.800 recursos digitales como: videos, podcast, módulos de formación; también se inició la trasmisión de programas educativos e informativos a través de radio y televisión, y para los estudiantes que no podían acceder a ninguno de estos recursos las fichas pedagógicas o guías del docente las cuales eran entregadas ya impresas, todo esto contando con el acompañamiento docente. 
Mientras tanto, (Gonzales, 2020), representante de la UNICEF en Ecuador, señala la importancia del acompañamiento docente durante esta pandemia, existe aproximadamente en el sector publico 168000 docentes, quienes han buscado diferentes maneras de mantener contacto con sus estudiantes y dar continuidad al proceso de enseñanza - aprendizaje, siendo el vínculo principal entre las familias y la escuela.

Hoy en día la educación gira en torno en la tecnología, tenemos estudiantes que son nativos digitales es decir que están constantemente relacionados con la tecnología utilizándolas como un medio para relacionarse, entretenerse, informarse y educarse, lo que ha sido de gran importancia durante este proceso de transición de la educación presencial a la educación a distancia; pero ¿Qué hay de los docentes? ¿Cuentan con la formación y las competencias necesarias para hacer uso de las tecnologías emergentes en el proceso educativa en este nuevo contexto de la pandemia?

Considerando todo lo abordado deriva el interés de analizar el uso de las tecnologías emergentes en la práctica educativa durante la pandemia.

\section{Referencial teórico}

Según la investigación realizada en Paraguay por (Picón et al. 2020), desarrollaron un estudio no experimental de campo descriptivo a 212 docentes, recolectando datos a través de cuestionario de Google Forms, sobre el Desempeño y formación docente en competencias digitales en clases no presenciales durante la pandemia COVID-19, con el objetivo de analizar el conocimiento y preparación de los docentes en las tecnologías de la información y comunicación (TIC) y su aplicación durante la pandemia; ya que a partir de la nueva modalidad educativa existe grandes desafíos e interrogantes sobre las competencias y conocimientos previos en el uso de tecnologías emergentes de los docentes y la manera en la que se está desarrollando la práctica docente durante la pandemia.

Los resultados obtenidos en esta investigación abordaron varios puntos como el hecho de que los docentes tuvieron que adecuar las practicas educativas tradicionales a las 
necesidades del contexto de la pandemia intentando mantener la misma experiencia que en clases presenciales, lo que produjo inconvenientes en el uso de plataformas digitales debido a problemas de conectividad y acceso a internet; los principales medios de comunicación usados fueron mensajes de texto a través de WhatsApp, redes sociales como Facebook, mensajería electrónica a través de los correos, y por ultimo las plataformas de videoconferencia las mismas que fueron usadas en un bajo porcentaje. En cuanto a las herramientas tecnológicas utilizadas en la práctica docente hay un alto nivel de uso del chat y de las plataformas de contenido audiovisual para la reproducción de video. Los resultados obtenidos en esta investigación indican la total falta de uso de herramientas de búsqueda, trabajo colaborativo, creación de contenido, plataformas de gestión de aprendizaje, siendo estas las menos utilizadas por los docentes, lo que infiere a la falta de preparación, conocimiento y competencias en el manejo de las distintas herramientas tecnológicas por parte de los docentes. Sin embargo, el rol de los docentes sigue siendo fundamental para el proceso de enseñanza-aprendizaje es por ello que surge la necesidad imprescindible de que sean capacitados en el manejo y aplicación de las TIC con el fin de proveer un aprendizaje eficaz.

Por otro lado, (Zunino, 2021), comenta que los conocimientos, destrezas, actitudes y estrategias con las cuales los docentes usan los medios digitales tiene gran relevancia en el proceso educativo ya que esto ayuda a mejorar la práctica docente siempre y cuando los recursos tecnológicos sean usados con un propósito educativo y no intentando suplantar la labor del docente. Para lograr lo antes mencionado es necesario que los docentes adquieran las competencias necesarias para que sean usadas de manera eficaz y optima en el proceso de enseñanza-aprendizaje.

\section{Tecnologías de la información y la comunicación aplicadas en la Educación}

Las Tecnologías de la Información y la Comunicación (TIC), son el conjunto de tecnologías que permiten acceder, crear, manejar, intercambiar y procesar información a 
través las diferentes herramientas digitales como la computadora, las tabletas, el teléfono, radio, televisión entre otros.

Para (De-la-Hoz-Franco, et al. 2019), las TIC son todos los instrumentos utilizados para manipular información en los distintos dispositivos electrónicos, aplicaciones, plataformas y redes a través de las que se puede generar, guardar, gestionar, procesa, y trasmitir recursos tecnológicos como textos, imágenes, videos y audio. En cuanto a (Hernández et al. 2019), sostienen que el termino TIC hace referencia a todo elemento tecnológico usado para generar, guardar y procesar información de distintos tipos.

Las TIC en el ámbito educativo ha generado gran relevancia puesto que no solamente se trata de la implementación de elementos tecnológicos tales como conexiones a internet, un proyector, una computadora entre otros; sino en la finalidad con la que estos elementos son incorporados en la práctica docente con criterios pedagógicos dejando de ser un simple instrumento para convertirse en un recurso didáctico con un fin educativo. Para la Organizacion de las Naciones Unidas para la Educación la Ciencia y la Cultura (UNESCO, 2019), la aplicación de las tecnologías de la información y comunicación son un complemento que puede enriquecer y transformar la educación. En base a esto se puede considerar el uso de las TIC como herramienta para el proceso de enseñanzaaprendizaje generando un nuevo escenario en donde se pueden mejorar los logros educativos de los alumnos implementando su aplicación a través de estrategias óptimas puede ser muy útil para mejora el currículo, la planificación, ejecución de las clases, el sistema de evaluación, y la práctica docente.

En otras palabras, podemos decir que las TIC aplicadas en la educación son todas aquellas tecnologías que engloban los equipos, sistemas y programas que favorecen a la adquisición, creación, procesamiento, intercambio, distribución y recepción de la información con fines educativos. Desde el punto de vista educativo considerando el aporte de (Galvis, 2004), las TIC se pueden clasificar en tres grupos según sus características: 
a) Los medios transmisivos, los cuales se utilizan para que el emisor envié el mensaje a los distintos receptores a través de recursos de video, audio, texto.

b) Los medios activos son los que permiten una actitud dinámica en el proceso educativo generando una experiencia enriquecedora mediante la reflexión como por ejemplo que son los que permiten generar un aprendizaje activo a través de recursos como traductores de idioma, herramientas de búsqueda.

c) Medios interactivos generando un aprendizaje constructivista de manera sincrónica o asincrónica con una participación a través de sistemas de mensajerías, juegos digitales, Foros, herramientas de trabajo colaborativo.

d) Las tecnologías emergentes nos brindan una serie de herramientas y recursos digitales que pueden ser utilizadas en el ámbito educativo en todos los procesos que esto implica: currículo, planificación, desarrollo de la clase, evaluación. Es necesario conocer la diferencia entre recurso digital y herramienta.

e) Los recursos digitales son los elementos que podemos encontrar en formato digital y a través de los distintos dispositivo electrónico como computadora, teléfono celular, radio y televisión entre otros.

Las herramientas digitales son todos aquellos recursos intangibles o inmateriales que no podemos ver, tocar o contar, en esta clasificación se encuentran todos los sistemas, aplicaciones, softwares en los cuales realizamos actividades a través de la tecnología cumple con un propósito; los recursos educativos digitales se convierten en un recurso didáctico digital cuando tiene intencionalidad educativa facilitando las estrategias de proceso de enseñanza- aprendizaje.

De acuerdo con (Oliveira \& Santos, 2021), las tecnologías emergentes se pueden determinar como un instrumento eficaz como apoyo para el proceso de enseñanza aprendizaje. Hoy en día los docentes deben involucrar las TIC en su metodología, pedagogía y estrategias educativas innovando la forma de impartir sus clases a través de los distintos recursos y herramientas digitales; para ello es necesario desarrollar las 
competencias necesarias por parte de los docentes a través de su formación y capacitación en el manejo y articulación de Los elementos tecnológicos dentro del sistema educativo.

Por otra parte la (Organización de Estados Iberoamericanos para la Educación, 2018), señala que en Ecuador el $54,86 \%$ de docentes poseen formación académica con relación al manejo de las TIC, ahora bien es necesario saber el uso que se le está dando dentro del contexto de la pandemia, puesto que las situaciones han cambiado y ahora el uso de los recursos tecnológicos es fundamental para desarrollar la práctica educativa tanto para docentes, estudiantes y todos los miembros que son parte de la comunidad Educativa.

\section{Sistematización de herramientas de apoyo para la práctica educativa}

Hoy en día encontramos un gran variedad de herramientas y recursos tecnológicos a los cuales podemos acceder, sin embargo debido a la falta de conocimiento de la existencia de estos recursos la practica educativa en la virtualidad se imparte en base a los recursos tradicionales que se aplicaban en clases presenciales, es por ello que mediante la siguiente clasificación se pretende ampliar la perspectiva de los docentes sobre algunas herramientas digitales que podrían aportar a la práctica educativa. 

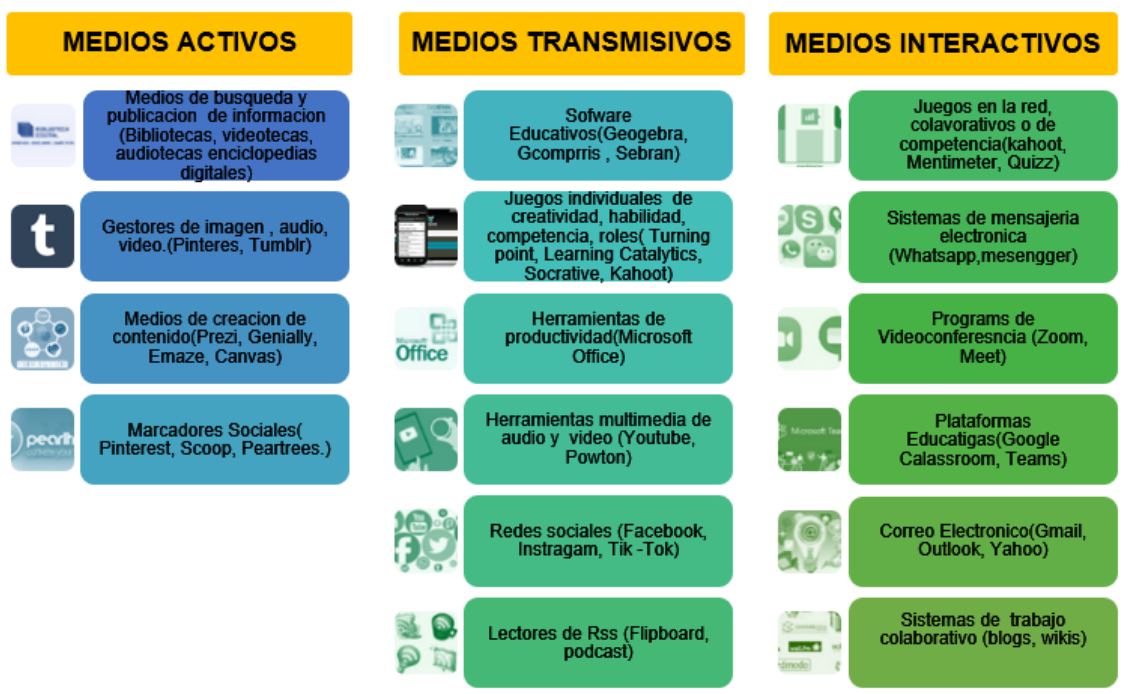

Figura 1. Recursos Educativos clasificados por características. Elaboración: Los autores.

Correo electrónico: medio que permite establecer una relación asíncrona, mediante la trasmisión de texto en el cual se puede adjuntar archivos de audio, video e imágenes.

Foros: espacios abiertos en donde los integrantes pueden generar temas de discusión en el que todos los participantes pueden intervenir.

Chat y Redes sociales: sistemas de intercambio de mensajes de texto, imágenes, videos y audio en el cual se puede establecer un medio de comunicación sincrónico o asincrónico.

Videoconferencia: medio de comunicación sincrónico que permite establecer un contacto visual y auditivo a través de la distancia.

Herramientas de trabajo colaborativo: medios que permiten la interacción entre docente y alumnos, mediante el uso compartido de distintos recursos creando espacios educativos.

Herramientas de búsqueda de información: medios que permiten recopilar información a través de los distintos navegadores y bibliotecas electrónicas. 
Lectores de RSS: medio que reúne información por categorías de distintas páginas web mediante la suscripción.

Herramientas Office: recopilación de herramientas que facilitan la creación, modificación de documentos en distintas formas, como documentos, bases de datos, presentaciones. Herramientas de creación de contenidos: medios que permiten crear, modificar y guardar, imágenes, audio o video.

Plataformas de gestión de aprendizaje: sitio web que engloba distintas herramientas como foros, wikis, presentaciones, espacios de recepción de tareas entre otros.

Espacios de administración de archivos digitales: medios que permiten administrar, organizar y guardar información de todo tipo.

Sistemas de respuesta en tiempo real: espacios que permiten la creación de contenidos mediante presentaciones o formularios que permiten la interacción de los participantes. Plataformas de contenido audiovisual: medio en el cual podemos encontrar información de tipo visual y auditivo.

Herramientas de creación de cuestionarios: medios que permiten la creación de cuestionarios y formularios en línea.

\section{Formación Docente en el uso de las TIC}

Hoy en día se ha incorporado las tecnologías emergentes en este nuevo escenario educativo de la virtualidad que para muchos docentes fue un nuevo reto que se asumió como otro rol docente con el fin de mejorar los procesos de formación educativa, se ha mencionado términos como competencias digitales que no es más que el conjunto de destrezas, estrategias y saberes adecuados para el uso de las tecnologías emergentes, las cuales se adquiere a través de la formación y la experiencia.

Por otro lado, (Valdivieso \& Gonzáles, 2016), indican que no solamente bastas con tener conocimientos sobre los aspectos técnicos de las TIC sino también habilidades y capacidades que los interrelacionen con los aspectos pedagógicos. En este sentido, se debería realizar un ajuste empezando por las metodologías tradicionales y las técnicas 
pedagógicas con las cuales los docentes han sido formados, así mismo, (Cobos-Velasco, et al. 2019), refiere que los docentes deben aprender a impartir conocimientos de una manera distinta dejando de lado los métodos habituales a través de las distintas herramientas tecnológicas. Por lo tanto, es necesario incluir las tecnologías de la información y la comunicación en la práctica educativa va más allá de brindar la formación adecuada a los docentes, ya que es un proceso que necesita de capacitación e innovación constante.

Para conseguir el uso adecuado de los distintos medios tecnológicos en la labor pedagógica a más de los conocimientos previos el docente debe tener actitudes y aptitudes favorables para incorporar las TIC. En el análisis realizado por (Reyes et al., 2018), genera gran connotación entre el empleo de las TIC y las competencias digitales que deben adquirir los docentes para desarrollar clases innovadoras, asumiendo que no solamente es necesario tener conocimientos sobre las herramientas digitales, sino que también es necesario saber cuándo, cómo y en qué momento utilizarlas durante el proceso enseñanza - aprendizaje para crear ambientes que motiven a los estudiantes con situaciones en las que los alumnos construyan sus conocimientos a través de las distintas herramientas y recursos tecnológicos, finalmente concluye en la necesidad de formación continua para los docentes en el contexto pedagógico y tecnológico a través de las siguientes estrategias:

- Fomentar programas que capaciten a los miembros de la comunidad educativa, en el uso de las TIC.

- Incorporar las herramientas y recursos tecnológicos accesibles en el proceso educativo.

- Motivar el uso de los distintos medios tecnológicos como mediadores de aprendizaje 
Revista Arbitrada Interdisciplinaria KOINONIA

Año VI. Vol VI. N³. Edición Especial: Educación II. 2021

Hecho el depósito de Ley: FA2016000010 ISSN: 2542-3088

FUNDACIÓN KOINONIA (F.K). Santa Ana de Coro. Venezuela.

Katherine Michelle Zhindón-Calle; Carlos Marcelo Ávila-Mediavilla

\section{Según el Marco Común de Competencia Digital Docente de España (Instituto Nacional de Tecnologías Educativas y Formación del Profesorado, 2017), plantea algunas áreas y competencias que el docente debe desarrollar.}

\section{Tabla 1.}

\section{Áreas y competencias digitales que el docente debe desarrollar.}

\begin{tabular}{ll}
\hline Área & Competencia \\
\hline $\begin{array}{l}\text { Información y alfabetización } \\
\text { informacional }\end{array}$ & $\begin{array}{l}\text { Identificar, localizar, obtener, almacenar, organizar y analizar información } \\
\text { digital, evaluando su finalidad e importancia }\end{array}$ \\
Comunicación y colaboración & $\begin{array}{l}\text { Interacción mediante tecnologías digitales. } \\
\text { Compartir información y contenidos. } \\
\text { Participación ciudadana en línea. } \\
\text { Colaboración mediante canales digitales. } \\
\text { Gestión de la identidad digital }\end{array}$ \\
Creación de contenidos digitales: & $\begin{array}{l}\text { Desarrollo de contenidos digitales nuevos. Integración y reelaboración de } \\
\text { contenidos digitales. } \\
\text { Realizar producciones artísticas, contenidos multimedia } \\
\text { Aplicar los derechos de autor y licencias de uso }\end{array}$ \\
& $\begin{array}{l}\text { Protección de información y datos personales, protección de la identidad } \\
\text { digital, medidas de seguridad, uso responsable y seguro. }\end{array}$ \\
& Resolución de problemas técnicos. \\
Identificación de necesidades y respuestas tecnológicas. \\
Innovación y uso de la tecnología digital de forma creativa
\end{tabular}

Fuente: Instituto Nacional de Tecnologías Educativas y Formación del Profesorado (2017). 
Como se visualiza en la tabla 1 hay varios aspectos que se deben tomar en cuenta en la formación docente para el uso de las TIC con el objetivo de adquirir competencias digitales necesarias para integrarlos de manera eficaz en el contexto educativo. El Ministerio de Educación de Ecuador elaboro la Agenda Educativa Digital como estrategia para la inserción de las TIC como un proceso de innovación para la educación a través de distintos lineamientos para mejorar la calidad educativa con el fin de dejar atrás la escuela tradicional y transformarla a una escuela digital.

Algunos de los objetivos planteados son promover la integración y el uso de las tecnologías de la información y la comunicación en el sistema educativo teniendo en cuenta las particularidades de los grupos más vulnerables, optimizar las competencias y capacidades de los ecuatorianos a través de programas de alfabetización digital, con el fin de disminuir la brecha tecnológica.

Algunos de los planes de Estrategia Ecuador digital desde el 2007 hasta el 2015 fueron: el Plan Nacional de Conectividad Escolar, la Creación de Infocentro y aulas móviles. La capacitación rural en tecnología (C.R.T.), con las cuales se equipó algunas instituciones y se potenció el uso de medios tecnológicos con fines educativos. El enfoque actual de la Agenda Educativa digital contempla cinco ejes:

1. Eje Físico: en cual se plantea la implementación de conexión a banda ancha para uso de docentes y estudiantes en todas las instituciones educativas a través de cuatro fases; además del equipamiento de las instituciones a través de laboratorios, laptop para el docente, parlantes y proyectores para cada aula.

2. Eje de Aprendizaje digital: pretende obtener un nuevo currículo actualizado con la incorporación de las tecnologías de la información, enlazando los componentes tradicionales del currículo con las TIC, mediante la creación del Currículo en línea que será un sistema web en el que se pueda vincular el currículo a las planificaciones micro curriculares y recursos digitales generando una red social dirigida a los docentes, al Igual que incorporar en la malla curricular el área de Ciencias de la Computación. 
Otro de los componentes de este eje Es la metodología pedagógica, en la cual se propone ejes transversales que contienen conceptos y teorías pedagógicas que generen una propuesta educativa innovadora. Los contenidos establecidos en el currículo se deben considerar como orientaciones, ya que es el docente quien está encargado de generar, reutilizar contenidos a más de los convenios con otras instituciones de educación superior que se encargan de la creación material educativo. Recursos Educativos Digitales que son parte de la propuesta educativa contempla todos aquellos que tengan un fin pedagógico o didáctico.

3. Eje de Desarrollo Docente: toma en cuenta a los docentes que no tuvieron formación en el uso de las TIC y a los futuros docentes que están en proceso educativos, a través de estrategias que faciliten el desarrollo de las competencias digitales y la integración de la tecnología en todo el proceso educativo como la planificación, desarrollo de la clase, guías metodológicas, todo esto a través de cuatro fases, en la primera el docente debe aprender y adquirir los fundamentos básicos sobre las TIC.

En la segunda deben aplicar los conocimientos adquiridos para adquirir experiencia en el uso de las mismas para mejorar el desempeño docente y generar clases más innovadoras, la tercera fase es incorporar las TIC a través del currículo generando proyectos de investigación dentro de las aulas, ya que para esta fase las aulas deben estar dotadas de conectividad y equipamiento; y por ultimo al incorporar los recursos tecnológicos en el proceso de enseñanza aprendizaje, renueva la practica educativa por ende sus estrategias para lograr aprendizajes colaborativos y significativos.

La formación continua es uno de los ejes fundamentales dentro de este aplicativo, puesto que busca una actualización permanente de conocimientos para poder integrarlos en las competencias digitales y pedagógicas de los docentes a través de cursos de formación con menor tiempo de duración y contenidos dosificados.

La formación inicial docente es un reto para las instituciones formadoras ya que de aquí deriva el desempeño de cada docente y la calidad de educación que brinde en base a los conocimientos y prácticas que adquirió en este proceso, se espera profesionales 
preparados, innovadores capaces de dar respuesta a los problemas actuales y afrontar las situaciones que día a día se presentan, en este sentido no solamente hablamos del perfil profesional del docente sino de calidad y calidez de persona que también se necesita en este ámbito.

4. Eje de comunicación y fomento: este eje pretende dar a conocer las implementaciones y los puntos mencionados con anterioridad, así como los ejes de esta agenda en todas sus fases con el fin de alcanzar mayor difusión y conocimiento como aporte para la sociedad.

5. Eje de innovación: con la implementación de los recursos tecnológicos pretende mejorar la calidad educativa, a través de la transformación de instituciones tradicionales a centros equipados con laboratorios, bibliotecas y recursos tecnológicos, en el ámbito de los docentes pretende generar un nuevo rol en donde desarrollen habilidades y estrategias para que puedan incorporar las tecnologías emergentes en su labor educativa.

Como podemos observar a más de la implementación de las distintas herramientas y recursos tecnológicos, la formación del docente y las competencias digitales adquiridas, así como la actualización recurrente en este proceso son fundamentales para transformar la practica educativa.

\section{MÉTODO}

La presente investigación se llevó a cabo en la Escuela de Educación Básica Gabriela Mistral en la ciudad de Cuenca, provincia del Azuay - Ecuador, se tomó como universo total de la población a los 24 docentes de la unidad educativa, y mediante muestreo aleatorio estratificado a 144 estudiantes de básica media y superior. Siendo una investigación de tipo descriptivo no experimental, por cuanto no hubo intervención en las variables y de cohorte transversal por que los datos obtenidos se recopilaron en un solo tiempo, con un paradigma epistemológico cuantitativo. 
Se aplicó una encuesta online mediante cuestionario que consta de 26 ítems en escala de valoración Likert tanto a docentes como estudiantes, fue validado por el método Delphi con el asentimiento de los distintos expertos como lo menciona (López-Gómez, 2018), mientras que la fiabilidad de las respuestas a través del coeficiente de correlación alfa de Cronbach mediante el software SPSS, dando como resultado en la encuesta aplicada a los docentes el 0.796 de fiabilidad mientras que en la encuesta ejecutada a los estudiantes se obtuvo el 0,910. Los datos obtenidos fueron procesados mediante estadistica descriptiva.

\section{RESULTADOS}

Se expondrá los resultados de las preguntas planteadas a docentes y estudiantes de la Escuela de Educación Básica Gabriela Mistral los mismos que fueron analizados en el software SPSS, aplicando la prueba de normalidad mediante Shapiro Wilk para las preguntas a los docentes y mediante Kolmogorov a los estudiantes, dando como resultado que todas las variables son paramétricas y estan asociadas.

\section{Tabla 2.}

Capacitación en la formación docente para el uso de las TIC.

\begin{tabular}{llcccc}
\hline & & Frecuencia & Porcentaje & Porcentaje válido & Porcentaje acumulado \\
\hline Válidos & Nunca & 4 & 16,7 & 16,7 & 16,7 \\
& Casi Nunca & 2 & 8,3 & 8,3 & 25,0 \\
& En Ocasiones & 12 & 50,0 & 50,0 & 75,0 \\
& Casi siempre & 5 & 20,8 & 20,8 & 95,8 \\
& Siempre & 1 & 4,2 & 4,2 & 100,0 \\
& Total & 24 & 100,0 & 100,0 & \\
\hline
\end{tabular}

Fuente: Encuesta. 
En la tabla 2 se puede observar que $4(16,7 \%)$ docentes indican que nunca recibieron capacitación sobre el uso de las TIC en su formación docente, $2(8,3 \%)$ casi nunca, mientras que $12(50 \%)$ en ocasiones, $5(20,8 \%)$ casi siempre y $1(4 \%)$ siempre, dando un total de $100 \%$, parte del objetivo de esta investigación es mejorar el uso de los recursos tecnológicos en la práctica educativa mediante la capacitación de los docentes.

\section{Tabla 3.}

Relación entre las clases mediante nuevos recursos tecnológicos y los docentes están preparados para usar recursos tecnológicos.

\begin{tabular}{|c|c|c|c|c|c|}
\hline \multirow{6}{*}{$\begin{array}{l}\text { Innovación de clases } \\
\text { mediante nuevos recursos } \\
\text { tecnológicos }\end{array}$} & \multirow{6}{*}{$\begin{array}{l}\text { Casi Nunca } \\
\text { En Ocasiones } \\
\text { Casi Siempre } \\
\text { Siempre }\end{array}$} & \multicolumn{3}{|c|}{$\begin{array}{l}\text { Los docentes están preparados para usar recursos } \\
\text { tecnológicos durante la pandemia }\end{array}$} & \multirow[b]{2}{*}{ Total } \\
\hline & & Casi Nada & \multirow{2}{*}{$\begin{array}{c}\text { Poco } \\
0\end{array}$} & \multirow{2}{*}{$\begin{array}{l}\text { Mucho } \\
0\end{array}$} & \\
\hline & & 1 & & & 1 \\
\hline & & 0 & 6 & 3 & 9 \\
\hline & & 0 & 5 & 5 & 10 \\
\hline & & 0 & 2 & 2 & 4 \\
\hline \multirow[t]{2}{*}{ Total } & & 1 & 13 & 10 & 24 \\
\hline & & Valor & \multicolumn{3}{|c|}{ Sig. asintótica (bilateral) } \\
\hline \multicolumn{2}{|l|}{ Chi-cuadrado de Pearson } & $24,646^{a}$ & \multicolumn{3}{|c|}{, 000} \\
\hline \multicolumn{2}{|l|}{ Razón de verosimilitudes } & 8,941 & \multicolumn{3}{|c|}{,177 } \\
\hline \multicolumn{2}{|l|}{ Asociación lineal por lineal } & 2,650 & \multicolumn{3}{|c|}{104} \\
\hline \multicolumn{2}{|l|}{ N de casos válidos } & 24 & & & \\
\hline
\end{tabular}

Fuente: Encuesta.

En la tabla 3 se puede notar que el valor de Sig. Bilateral para la Chi cuadrado de pearson es menor que 0,05 , lo que demuestra que es una hipótesis afirmativa( $\mathrm{H} 1)$, ya que existe una relación entre la preparación de los docentes en el uso de los recursos tecnológicos y la innovación de las clases a través de las TIC, lo que apunta a que mediante una 
Revista Arbitrada Interdisciplinaria KOINONIA

Año VI. Vol VI. N³. Edición Especial: Educación II. 2021

Hecho el depósito de Ley: FA2016000010 ISSN: 2542-3088

FUNDACIÓN KOINONIA (F.K). Santa Ana de Coro. Venezuela.

Katherine Michelle Zhindón-Calle; Carlos Marcelo Ávila-Mediavilla

capacitacion en el uso herramientas y recursos tecnologicos nos ayudara a generar espacios de aprendizaje mas favorables.

Tabla 4.

Relación entre las variables el docente ha usado recursos tecnológicos innovadores y las Clases son innovadoras.

\begin{tabular}{|c|c|c|c|c|c|c|}
\hline \multirow{5}{*}{$\begin{array}{l}\text { El docente ha usado } \\
\text { recursos tecnológicos } \\
\text { innovadores }\end{array}$} & \multirow[b]{3}{*}{ Nunca } & \multicolumn{4}{|c|}{ Las Clases son innovadoras } & \multirow[b]{2}{*}{ Total } \\
\hline & & \multirow{2}{*}{$\begin{array}{c}\text { Nunca } \\
3\end{array}$} & \multirow{2}{*}{$\begin{array}{c}\text { Casi Nunca } \\
0\end{array}$} & \multirow{2}{*}{$\begin{array}{c}\text { Casi Siempre } \\
2\end{array}$} & \multirow{2}{*}{$\begin{array}{c}\text { Siempre } \\
1\end{array}$} & \\
\hline & & & & & & 6 \\
\hline & Casi Nunca & 1 & 19 & 14 & 19 & 53 \\
\hline & Casi Siempre & 0 & 7 & 11 & 17 & 35 \\
\hline & Siempre & 0 & 3 & 9 & 44 & 56 \\
\hline \multirow[t]{2}{*}{ Total } & & 4 & 29 & 36 & 81 & 150 \\
\hline & & \multicolumn{2}{|l|}{ Valor } & Sic & \multicolumn{2}{|c|}{ Sig. asintótica (bilateral) } \\
\hline \multicolumn{2}{|l|}{ Chi-cuadrado de Pearson } & \multicolumn{2}{|l|}{$81,114^{a}$} & 9 & \multicolumn{2}{|c|}{, 000} \\
\hline \multicolumn{2}{|l|}{ Razón de verosimilitudes } & \multicolumn{2}{|l|}{47,328} & 9 & \multicolumn{2}{|l|}{, 000} \\
\hline \multicolumn{2}{|l|}{ Asociación lineal por lineal } & \multicolumn{2}{|l|}{27,147} & 1 & \multicolumn{2}{|l|}{, 000} \\
\hline N de casos válidos & & \multicolumn{2}{|l|}{150} & & & \\
\hline
\end{tabular}

a. 7 casillas $(43,8 \%)$ tienen una frecuencia esperada inferior a 5 . La frecuencia mínima esperada es ,16.

Fuente: Encuesta.

En la tabla 4 se puede notar que el valor de Sig. Bilateral para la Chi cuadrado de pearson es menor que 0,05 , por lo que se asume que el uso de recursos tecnologicos innovadores esta relacionado en gran medida con una clase innovadora, esto desde el punto de vista de los estudiantes. 

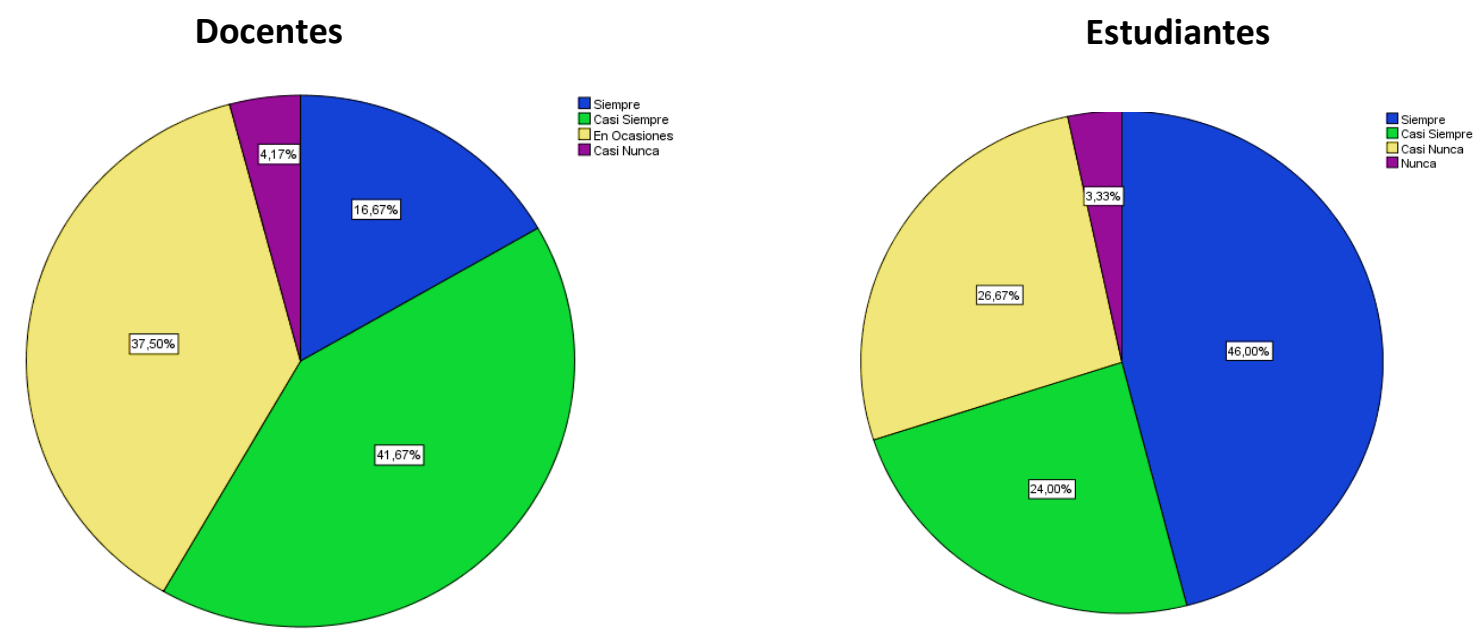

Figura 2. Comparación nivel de empleo de recursos tecnológicos innovadores durante la pandemia perspectiva de docente y estudiantes.

Fuente: Encuesta.

Mediante esta figura podemos observar la perspectiva de los docentes y estudiantes en cuanto al uso de recursos tecnológicos innovadores durante las clases, obteniendo una respuesta favorable por parte de los estudiantes, puesto que la mayoría considera que los recursos utilizados en este periodo de la pandemia han sido innovadores. 


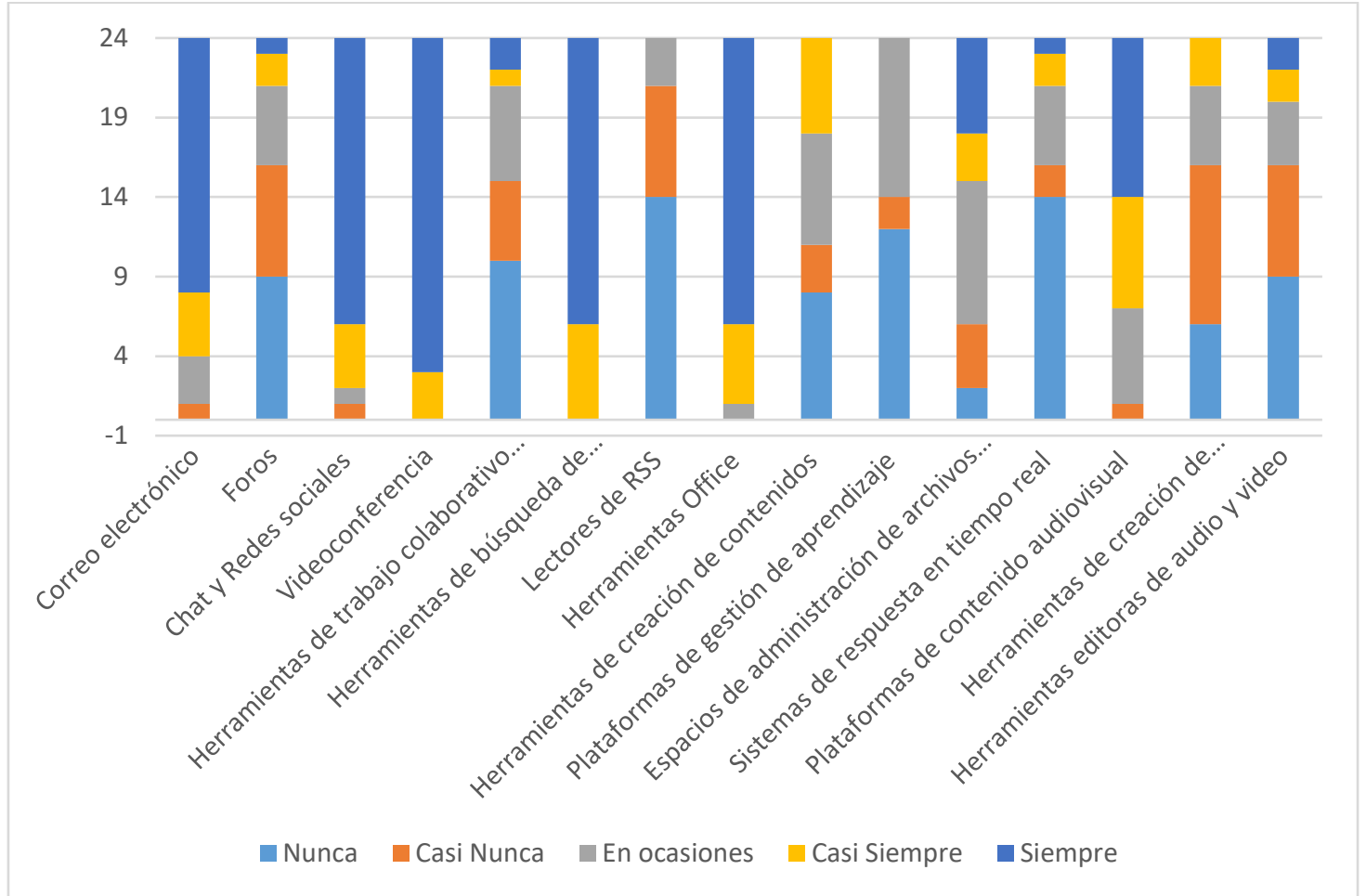

Figura 3. Recursos Tecnológicos usados por los docentes.

Fuente: Encuesta.

En la figura 3 se puede observar los recursos tecnologicos usados por los docentes con mas frecuencia, lo que nos hace conciderar que ha pesar de no haber estado preparados para la implementacion de las TIC en un contexto virtual han buscado la manera de adquirir conociemiento en uso y aplicación de distintos recursos tecnologicos para mejorar la practica educativa a traves de la distancia.

A concecuencias de las medidas implementadas durante la pandemia por el COVID-19 se ha podido considerar el uso de las tecnologías emergentes como uno de los principales medios de educación a través de las distintas herramientas y recursos educativos que podemos encontrar, sin desmerecer el esfuerzo de los docentes para sobrellevar la situación con la búsqueda constante de nuevas estrategias con el objetivo de generar aprendizajes significativos involucrando a los estudiantes para despertar su interés. Coincidiendo con (Muñoz-Mejía et al. 2020), con que la motivación en el 
aprendizaje es fundamental, y esto se puede lograr a través del uso de distintos recursos interactivos; para esto es necesario la incorporación de herramientas y recursos distintos a los que se usaba en las clases presenciales.

\section{PROPUESTA}

Basándonos en los resultados obtenidos de las encuestas realizadas se puede notar que los cambios a los cuales estamos sujetos por la pandemia COVID-19, ha generado que los docentes y estudiantes tengan que hacer uso de los distintos recursos tecnológicos en su práctica educativa diaria, buscando la manera de innovar esta actividad.

La herramienta Quizziz es un medio de aprendizaje con el cual se puede potenciar la comprensión y habilidades de los estudiantes para obtener aprendizajes significativos.

Es por ello que nos orientamos a plantear la siguiente propuesta de capacitación a los docentes sobre la incorporación y el uso de este elemento con el fin de generar un mayor impacto en el proceso de enseñanza aprendizaje a través de los sistemas de respuesta en tiempo real como Quizziz mediante el cual los docentes pueden generar presentaciones de manera creativa con animaciones e interacciones interesantes, formulación de cuestionarios que se pueden asignar como tareas o responderla en tiempo real y también se puede encontrar un conjunto de cuestionarios los cuales son reutilizables.

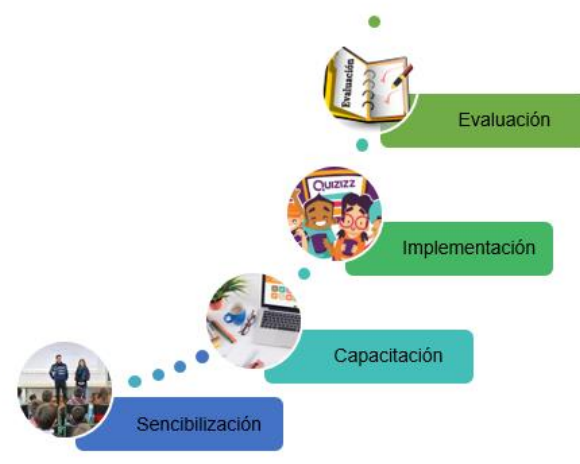

Figura 4. Estrategia de capacitación para el uso de Quizziz. Elaboración: Los autores. 
Sensibilización. - Sobre la importancia de actualizar sus conocimientos en relación a los distintos recursos tecnológicos que pueden ser usados con fines educativos con el objetivo de mejorar la práctica docente.

Capacitación. - Se puede ingresar a la nueva herramienta mediante el sitio web o la aplicación, es una herramienta de acceso gratuito, el primer paso es realizar el registro dentro de la aplicación mediante el correo de Google u otro, al ingresar encontramos una serie de pestañas que nos permitirán acceder a la creación de cuestionarios y a un repositorio de formularios, que se pueden reutilizar.

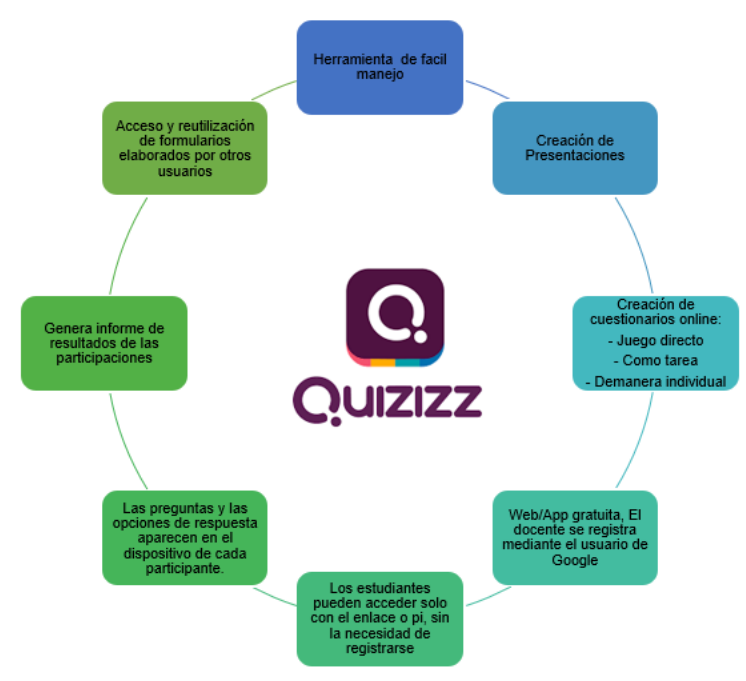

Figura 5. Elementos de la Herramienta Quizziz. Elaboración: Los autores.

Implementación.- Con el usuario registrado y los cuestionarios elaborados, se debe planificar y determinar el momento de la clase en el que se quiera aplicar esta herramienta, brindar a los estudiantes una breve explicación de los pasos a seguir para ingresar al formulario proporcionándoles el enlace de ingreso o el pin, de acuerdo a la opción que se haya elegido dentro del programa para la aplicación de la presentación o 
el cuestionario, el docente tiene la potestad de controlar el avance de las diapositivas o preguntas brindándoles un tiempo ya antes fijado para poder responder, al finalizar cada formulario se obtendrá un informe detallado con puntuaciones asignadas a cada usuario según las respuestas correctas y los tiempos de respuesta.

Evaluación. - A través de la evaluación podemos constatar el uso de esta herramienta digital y el impacto que ha generado a docentes y estudiantes, lo que no solo nos direcciona a comprobar su uso sino las innovaciones educativas por parte de los docentes al adquirir nuevos conocimientos y habilidades de manera creativa para mejorar sus competencias digitales.

\section{CONCLUSIONES}

La capacitación en cuanto a las TIC con fines educativos debería ser fundamental en la formación docente, ya que vivimos en un mundo de constantes cambios mediados por la tecnología, la pandemia COVID-19 ha impulsado el uso de los medios tecnológicos en los procesos de enseñanza - aprendizaje, los docentes han cambiado su perspectiva acerca del uso de las TIC, ya no son visto como un apoyo dentro de la practica educativa sino como el medio idóneo para conseguir un fin pedagógico.

A través de los resultados obtenidos en la encuesta se puede mencionar que al menos la mitad de los docentes que participaron en esta investigación manifiestan que no tuvieron una formación pedagógica con la capacitación adecuada para usar y aplicar los distintos recursos tecnológicos y tampoco estaban preparados para el cambio radical de las clases presenciales a la virtualidad, sin embargo, para sobrellevar la situación se han visto en la obligación de optimizado esfuerzos por implementar distintas estrategias para dar continuidad al proceso educativo a través del uso de las distintas tecnologías emergentes con el objetivo de generar aprendizajes significativos en los estudiantes.

Si bien es cierto nuestros docentes no han desarrollado en su totalidad las competencias digitales, esto no ha sido un impedimento, más bien a servido de impulso para que se auto eduquen con la búsqueda constante de nuevas herramientas y recursos 
tecnológicos para brindar la motivación necesario a los estudiantes con el fin de que se involucren a un mas en el proceso de enseñanza - aprendizaje.

Los docentes tienen la predisposición para implementar en su practica docente herramientas y recursos tecnológicos que brinden la oportunidad de generar clases mucho más dinámicas e innovadoras es por ello que se plantea la propuesta anterior con el objetivo de capacitar a los docentes para que adquieran nuevas conocimientos y a su vez puedan incorporar nuevas herramientas tecnológicas como el sistema de respuestas en tiempo real Quizás, ya que a través de ella puede generar espacios de aprendizaje didácticos que motiven a los estudiantes.

\section{FINANCIAMIENTO}

No monetario.

\section{AGRADECIMIENTO}

A la Jefatura de Posgrados de la Universidad Católica de Cuenca por permitir el desarrollo y fomento de la investigación.

\section{REFERENCIAS CONSULTADAS}

Cobos-Velasco, J. C., Jaramillo Naranjo, L. M., \& Vinueza Vinueza, S. (2019). Las competencias digitales en docentes y futuros profesionales de la Universidad Central del Ecuador [The Digital Competences in Teachers and Future Professionals of the Central University of Ecuador]. Cátedra, 2(1), 76-97. https://doi.org/10.29166/catedra.v2i1.1560

Constitución de la Republica del Ecuador 2008. Registro Oficial 449 de 20-oct-2008 Ultima modificación: 13-jul-2011. https://n9.cl/hd0g

De-Giusti, A. (2020). Policy Brief: Education during COVID-19 and beyond. Revista Iberoamericana De Tecnología En Educación Y Educación En Tecnología, (26), e12. https://doi.org/10.24215/18509959.26.e12 
De-la-Hoz-Franco, E., Martínez-Palmera, O., Combita-Niño, H., \& Hernández-Palma, H. (2019). Las Tecnologías de la Información y la Comunicación y su Influencia en la Transformación de la Educación Superior en Colombia para Impulso de la Economía Global [Information and Communication Technologies and their Influence on the Transformation of Higher Education in Colombia to Boost the Global Economy]. Información tecnológica, 30(1), 255262. https://dx.doi.org/10.4067/S0718-07642019000100255

Galvis, A. (2004). Oportunidades educativas de las TIC [Educational Opportunities of ICT]. Recuperado desde https://n9.cl/uyjnb

Gonzales, J. (2020). La educación en Ecuador tiene que continuar y todos debemos apoyarla, asegura Unicef [Education in Ecuador has to continue and we must all support it, says Unicef]. Recuperado desde https://n9.cl/b79xw

Hernández, R., Sánchez Cáceres, I, Zarate Hermoza, J, Medina Coronado, D, Loli Poma, T, \& Arévalo Gómez, G. (2019). Tecnología de Información y Comunicación (TIC) y su práctica en la evaluación educativa [Information and Communication Technology (ICT) and its Practice in Educational Evaluation]. Propósitos $y$ Representaciones, 7(2), 1-5. https://dx.doi.org/10.20511/pyr2019.v7n2.328

Instituto Nacional de Tecnologías Educativas y Formación del Profesorado. (2017). Marco Común de Competencia Digital Docente [Common Framework for Teaching Digital Competence]. https://n9.cl/olmz

López-Gómez, E. (2018). El método delphi en la investigación actual en educación: Una revisión teórica y metodológica [The Delphi Method in Current Research in Education: A Theoretical and Methodological Review]. Educación XX1, 21(1), 1740. https://doi.org/10.5944/educxx1.20169

MINEDUC. (2020). Plan Educativo COVID-19 [COVID-19 Educational Plan]. https://educacion.gob.ec/plan-educativo-covid-19/

Muñoz-Mejía, B., García-Herrera, D., Guevara-Vizcaíno, C., \& Erazo-Álvarez, J. (2020). Innovación docente en espacios virtuales y aplicación de WIX en el aula [Teaching innovation in virtual spaces and application of WIX in the classroom]. EPISTEME KOINONIA, 3(1), 4-24. http://dx.doi.org/10.35381/e.k.v3i1.989 
Revista Arbitrada Interdisciplinaria KOINONIA

Año VI. Vol VI. N³. Edición Especial: Educación II. 2021

Hecho el depósito de Ley: FA2016000010

ISSN: $2542-3088$

FUNDACIÓN KOINONIA (F.K). Santa Ana de Coro. Venezuela.

Katherine Michelle Zhindón-Calle; Carlos Marcelo Ávila-Mediavilla

Oliveira, A. de, \& Santos, P. (2021). The use of technological resources in teacher training as a contribution to teaching statistics, probability and combinatorial analysis at the National Meeting of Mathematical Education in Brazil. International Journal of Engineering, Management and Humanities (IJEMH), 2(1), 109-121.

Organización de Estados Iberoamericanos para la Educación, la C. y la C. (OEI), Instituto de Evaluación (IESME) de la OEI, \& Fundacion Telefonica. (2018). Estudio sobre la inclusión de las TIC en los centros educativos de aulas fundacion electronica. [Study on the inclusion of ICT in the educational centers of the electronic foundation classrooms]. https://n9.cl/29wkw

Organizacion de las Naciones Unidas para la Educación la Ciencia y la Cultura, UNESCO (2012). Las tic en la educación. [ICT in education]. https://es.unesco.org/themes/tic-educacion

Palomeque, M., \& Parrales, M. (2021). La resiliencia y satisfacción laboral de docentes en una institución educativa de la ciudad de Guayaquil [Resilience and job satisfaction of teachers in an educational institution in the city of Guayaquil]. 593 Digital Publisher CEIT, 6(1), 303-317. https://doi.org/10.33386/593dp.2021.1.427

Picón, G., González, G., \& Paredes, J. (2020). Desempeño y formación docente en competencias digitales en clases no presenciales durante la pandemia COVID-19. [Performance and Teacher Training in Digital Competences in Non-Presential Classes During the COVID-19 Pandemic]. Universidad Privada María Serrana, Asunción, 1-16. https://doi.org/https://doi.org/10.1590/SciELOPreprints.778

Reyes, J., Cardenas, M., \& Díaz, E. (2018). Las Competencias Digitales: una necesidad del docente Ecuatoriano del siglo XXI [A Need for the Ecuadorian Teacher of the XXI Century]. Revista Dilemas Contemporáneos: Educación, Política y Valores, Edición Especial, año VI, 1-17.

Singh, V., \& Thurman, A. (2019). How Many Ways Can We Define Online Learning? A Systematic Literature Review of Definitions of Online Learning (1988-2018). American Journal of Distance Education, 33(4), 289-306. https://doi.org/10.1080/08923647.2019.1663082

Valdivieso, T., \& Gonzáles, M. A. (2016). Competencia Digital Docente: ¿Dónde Estamos?. Perfil Del Docente De Educación Primaria y Secundaria. El Caso De Ecuador. [Teaching Digital Competence: Where are we? Profile Of The Teacher Of Primary And Secondary Education. The Case of Ecuador]. Píxel-Bit, Revista de Medios y Educación, 49, 57-73. http://hdl.handle.net/11441/45210 
Revista Arbitrada Interdisciplinaria KOINONIA

Año VI. Vol VI. N³. Edición Especial: Educación II. 2021

Hecho el depósito de Ley: FA2016000010 ISSN: 2542-3088

FUNDACIÓN KOINONIA (F.K). Santa Ana de Coro. Venezuela.

Katherine Michelle Zhindón-Calle; Carlos Marcelo Ávila-Mediavilla

Zunino, E. (2021). Medios digitales y COVID-19: sobreinformación, polarización y desinformación [Digital Media and Covid-19: overinformation, polarization and misinformation]. Universitas, Revista de Ciencias Sociales y Humanas, (34), 133154. https://doi.org/10.17163/uni.n34.2021.06

@2021 por los autores. Este artículo es de acceso abierto y distribuido según los términos y condiciones de la licencia Creative Commons Atribución-NoComercial-Compartirlgual 4.0 Internacional (CC BY-NC-SA 4.0)

(https://creativecommons.org/licenses/by-nc-sa/4.0/). 